\title{
Restoration of primary molars in a specialist paediatric dental practice
}

\author{
The survival of resin modified glass ionomer and stainless steel crown restorations in primary molars, placed in a \\ specialist paediatric dental practice
}

J. F. Roberts, N. Attari and M. Sherriff Br Dent J 2005; 198: 427-431

\section{Aims}

To prospectively report on the survival of resin-modified glass ionomer cement (RMGIC), photac-fil and pre-formed stainless steel crown (SSC) restorations in primary molar teeth placed over a seven-year period in a specialist paediatric dental practice under private contract of remuneration.

\section{Method}

All primary molar restorations placed by a specialist paediatric dentist over a seven-year period were reviewed and the outcome results recorded. Data were recorded at review visits until June 30, 2003. Data recorded included Class I restorations, Class II restorations and SSC. The Class II cavities were either mesial or distal, with or without buccal/palatal extensions. If both proximal surfaces were decayed or if after cavity preparation the resultant outline form was significantly larger than the minimal classical form, RMGIC was not used; a SSC was placed instead. Stainless steel crown preparation followed conventional guidelines. The crowns were cemented with reinforced zinc oxide and eugenol (Kalzinol). The status was recorded as satisfactory restoration, tooth exfoliated, tooth extracted for orthodontic reasons with the date of extraction, or needing replacement. If replaced then the reason for replacement was also recorded.

\section{Results}

A total of 544 Class I RMGICs, 962 Class II RMGICs, and 1,010 SSCs were placed. At the last review of each restoration, 98.3\% of Class I, 97.3\% of Class II RMGICs and 97.0\% of SSCs were either satisfactory or withdrawn intact.

\section{Conclusion}

Under the conditions of private specialist practice-based study SSCs continued to prove very successful for the restoration of larger cavities and for pulp-treated primary molar teeth. For the smaller cavities RMGIC were also very successful.

\section{IN BRIEF}

- Under specialist practice conditions, resin-modified glass-ionomer cement (RMGIC) restorations are shown to be highly successful.

- Class II RMGICs were as successful as preformed metal crowns.

- Emphasis is placed upon only placing intra-coronal restorations in minimal cavities, preformed crowns being used where the decay process is more advanced.

- High usage of rubber dam reflects the authors' firmly held belief that not only does it provide moisture control, but aids considerably in behaviour management.

\section{COMMENT}

Management of caries in the primary dentition has been the subject of much debate recently. ${ }^{1}$ Discussion of this kind can only be helpful for the profession as it stimulates thinking and encourages further research. Prevention of caries is preferable but in practice, patients will often present with advanced carious lesions. This paper, looking at the survival of restorative materials in children, comes at an opportune moment.

Survival rates of resin modified glass ionomer (RMGIC) and stainless steel crown restorations (SSC) in primary molars were studied. This was not a comparison of these two materials, rather a prospective report of the success and failure of these materials when placed in small and large cavities respectively.

The sample size was large and the drop out rate acceptable. The study was carried out on a child population (mean age ranged from 5.8 to 7.5). Rubber dam was used in the majority of cases. Use of local anesthetic and general anaesthetic varied, approximately half of all Class I RMGIC and SSC restorations were carried out under general anaesthetic and $80 \%$ of Class II RMGICs were carried out under local anaesthetic. Excellent survival rates for all types of restoration were demonstrated with true failures ranging from $1.7 \%$ to $3 \%$. This demonstrates that it is possible to reliably and successfully restore the primary dentition.

Criticism could be made of the fact that there was only one operator, and that many restorations were carried out under general anaesthetic thus eliminating problems of cooperation etc. However, an equally large proportion of restorations were placed using local anaesthetic and considering that the overall failure rates were low, most of these must have been successful. It is also important to remember that techniques and materials used in this study are not the sole province of the specialist but are taught and examined at undergraduate level.

Milsom, Tickle and King describe the aim of the GDP to 'provide a level of dental care that will take a child through the primary and mixed dentition phase.' ${ }^{1}$ The approach taken by the author of this study would seem to do just that. There is still much we need to understand about provision of dental care for children, nevertheless if you had to choose between no treatment, or a restoration with a greater than $90 \%$ chance of success, which would you pick for your child?

\section{P. Ashley, Clinical Lecturer, Unit of Paediatric Dentistry Eastman Dental Institute for Oral Health Care Sciences, UCL doi: $10.1038 /$ sj.bdj.4812794}

1. Milsom K M, Tickle M, King D. Does the dental profession know how to care for the primary dentition? Br Dent J 2003; 195: 301 